\section{Anaesthesiological problems in children with congenital laryngeal stenosis}

\author{
BILJANA DRAŠKOVIĆ • ANNA URAM BENKA • DRAGAN DRAŠKOVIĆ • \\ DUŠAN MARIĆ • DUŠICA SIMIĆ
}

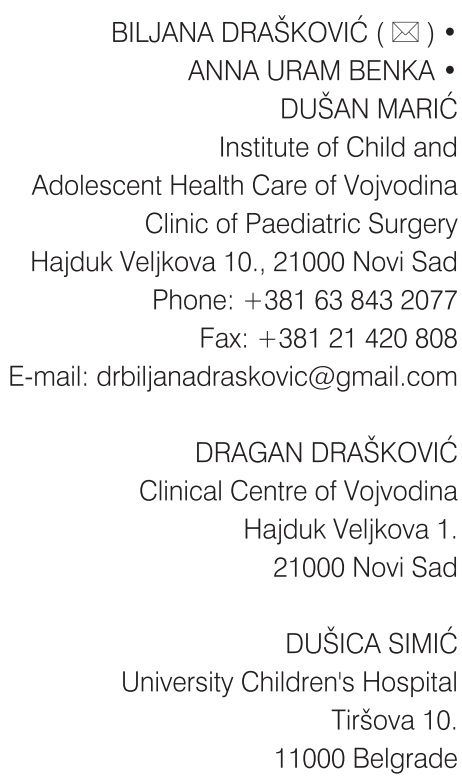

BILJANA DRAŠKOVIĆ $(\bowtie) \cdot$ ANNA URAM BENKA • DUŠAN MARIĆ Institute of Child and Adolescent Health Care of Vojvodina Clinic of Paediatric Surgery Hajduk Veljkova 10., 21000 Novi Sad Phone: +381638432077 Fax: +38121420 808 E-mail: drbiljanadraskovic@gmail.com

DRAGAN DRAŠKOVIĆ Clinical Centre of Vojvodina Hajduk Veljkova 1. 21000 Novi Sad

DUŠICA SIMIĆ University Children's Hospital Tiršova 10. 11000 Belgrade

\begin{abstract}
Congenital laryngeal stenosis is a rare condition, which can be diagnosed in the early neonatal period. Manifest stenosis can be life threatening. Severe ("critical") types of stenosis require securing an adequate airway just after the birth, as well as early tracheotomy as a life-saving procedure.

This paper presents a case report of a preterm newborn with severe laryngeal stenosis diagnosed at birth. It was not possible to secure the airway even with endotracheal tubes of the smallest diameter, thus a laryngeal mask (LMA) was placed to provide adequate ventilation and oxygenation. Anaesthesia was administered in the same way during the tracheotomy procedure, which was performed in the earliest hours of the baby's life.
\end{abstract}

Key words: laryngeal stenosis, laryngeal mask, newborn, airway

\section{Introduction}

Congenital laryngo-tracheal stenosis is a rare disease, which can be detected in the early neonatal period. This malformation can occur as an isolated manifestation or, more frequently, associated with other anomalies. $(1,2)$ In severe types of stenosis it is of paramount importance to secure an airway immediately after birth, and to perform an early tracheostomy so that an adequate airway can be temporarily secured until definite operative tracheal reconstruction, which can be done at a later stage. $(3,4)$ Preparation and formation of an urgent tracheostomy requires time, a skilled team of professionals and appropriate equipment. All this is usually not available next to the delivery room. Thus, if the newborn's life is endangered because of respiratory insufficiency, it is of vital importance to secure the airway by effective means available and artificial ventilation. (5)

\section{Case report}

A male newborn, from an in- vitro fertilization pregnancy, was delivered by Caesarean section in the $35^{\text {th }}$ week of gestation because of a prenatal diagnosis of bowel obstruction. At birth the newborn weighed $2140 \mathrm{~g}$ and the Apgar score was 4/5. Hypersalivation and moderate respiratory insufficiency were noticed. The newborn had tachycardia, tachypnoea and pale cyanotic skin. During the first few minutes of the child's life, upper airway aspiration was performed with $100 \%$ oxygen. Next, the patient was intubated, transferred to the intensive care unit (ICU), and submitted to artificial ventilation. As proper oxygenation and ventilation were still not achieved, and suspecting a tracheooesophageal fistula, the newborn was transferred to the Pediatric Surgical Unit. The tube position was rechecked prior to transfer, confirming the flow of air through the rima glotti- 
des. During transportation the baby was tachypneaic, so it was assisted by ventilating with $100 \%$ oxygen by a rebreeding bag. Transcutaneous saturation ranged from 90 to $92 \%$. Thoracic auscultation recorded only the sound of spontaneous breathing. After admission to the Pediatric Surgical Unit, a chest X-ray was performed, revealing a highly positioned endotracheal tube. This finding, as well as considerable "leakage" of insufflated air from the oropharynx, strongly suggested an unplanned extubation, thus both the laryngoscopy and the tube replacement were repeated. Throughout this period the newborn's skin was pale-pink in color, transcutaneous saturation was around $90 \%$ and cardiac rhythm was regular. Tachydyspnea with obvious tiredness while breathing was evident. Laryngoscopy revealed the epiglottis, an unusually wide larynx (for this age), and the glottides. Intubation with the No 3 tube failed, most likely due to a subglottic obstruction. Therefore, the procedure was repeated using a smaller tube, and yet without success. Behind the vocal cords there was a whitish barrier with the small opening. Since the passing of the smallest-diameter tube was not possible, the laryngeal mask (LMA) number 1 (The Laryngeal Mask Company Limited) was placed. Manual ventilation was started, resulting in visible elevation of chest cavity, adequate gas exchange, and soon better general condition of the baby was evident. Mechanical ventilation and full moni- toring were continued in the Intensive Care Unit (ICU), and because of the suspicion of tracheal stenosis an otorhinolaryngologist was consulted. After 15 minutes the child was taken to the operating room. Through the LMA the newborn was anesthetized with sevofluran (volatile induced maintenance anaesthesia-VIMA). Once adequate depth of anesthesia was achieved, the LMA was removed, and a rigid tracheobronchoscopy- Storz, of 2,5mm was placed, but came across a narrowing of $2 \mathrm{~mm}$. No neonatal bronchoscope could pass through such an opening, so the length of the narrowing was estimated using a bronchoscope. When the length of stenosis was estimated, the bronchoscope was removed and LMA placed back to continue ventilation during the tracheostomy. After the tracheostomy, a plastic uncuffed number 2 cannula was placed. Respiration continued via the tracheal cannula. Postoperative course was uneventful. Once the general condition of the baby was stable, investigations were continued. Prenatally diagnosed duodenal atresia was confirmed, whilst other organs were normal. On the second day of the baby's life, duodenal atresia was operated by resection and termino-terminal anastomosis. Postoperative period was normal.

After the tracheostomy, the parents were instructed on appropriate postoperative care, and the patient was dismissed from hospital in good general condition.

At the age of one the child underwent final treatment of tracheal stenosis and closing of the tracheostomy. Laryngotracheoplastics was carried out using the rib cartilage as grafting material. The tracheostomy was closed and the child was transnasally intubated for 7 days until the trachea healed. The child was given adequate analgesia and sedation in order to tolerate the endotracheal tube. When the general condition and the child's state of mind are good, analgesia and sedation present a great challenge for the anaesthesiologist. In this case we modified the strategies of analgesia and sedation by applying different medications (sufentanil, remifentanil, midazolam, and chloral-hydrate). During the first 24 hours after surgery the child was artificially ventilated and subsequently, adequate breathing via the endotracheal tube was enabled. Due to carefully titrated medications and continual monitoring, this stage of the patient's recovery was without complications.

On the eighth day of recovery a control bronchoscopy was performed, after which the child was extubated. The process of extubation was uneventful, so was further treatment. After three weeks of hospitalization, the child was discharged.

The main problems related to anaesthesiology in children with congenital respiratory anomalies are securing the airway after birth and achieving adequate analgesia and sedation after the reconstruction of the trachea. In this case both problems were successfully solved.

\section{REFERENCES}

1. Manson D, Filler R, Gordon R. Tracheal growth in congenital tracheal stenosis. Pediatr Radio 1996; 26:427-30.

2. Cotton RT, Prescott CJ . Congenital anomalies of the larynx. In: Cotton RT, Myer CM, editors. Practical pediatric otolaryngology. Philadelphia, PA: Lippincott-Raven; 1999. p. 497-514.

3. Anton-Pacheco J L, Cano I, Garcia A, Martínez A, Cuadros J , Berchi FJ . Patterns of management of congenital tracheal stenosis. J Pediatr Surg 2003;38(10):1452-8.

4. Wyatt ME, Hartley BJ . Laryngotracheal reconstruction in congenital laryngeal webs and atresias. Otolaryngol Head Neck Surg 2005;132:232-8.

5. Chen I, Hsaio AL. Randomized Trial of Endotracheal Tube versus Laryngeal Mask Airway in Simulated Prehospital Pediatric Arrest. Pediatrics 2008;122:294-97. 